\title{
Study Day of Healthcare Encounters
}

National Cancer Institute

\section{Source}

National Cancer Institute. Study Day of Healthcare Encounters. NCI Thesaurus. Code C123951.

The study day that a healthcare encounters assessment is performed. 